\title{
Bronchoscopy Safety Box and its Utility as a Barrier in Spread of COVID-19 Infection
}

\author{
Sameer A. Arbat, Sweta Raja Chourasia \\ Department of Pulmonary, Ketki Research Institute of Medical Sciences, Nagpur, Maharashtra, India
}

\section{Abstract}

Introduction: The COVID-19 pandemic has infected millions with a high mortality rate. Thousands of frontline health-care workers (HCWs) have been infected with SARS-CoV-2. Protecting HCWs is important. Aim: We sought to develop an innovative barrier to prevent COVID-19 virus transmission while performing bronchoscopy and endotracheal intubation. Settings and Design: We have designed and developed a barrier called bronchoscopy safety box (BSB). Materials and Methods: BSB is made up of acrylic and polyvinyl chloride (PVC) sheets. We have designed two prototypes of BSBs, namely Prototype 1 and Prototype 2. We performed bronchoscopy procedures and intubation procedures with BSBs. Advantages and limitations were noted and analyzed. Results: The advantages of BSB include the additional presence of the bronchoscopy aperture and an assistant aperture. All the apertures are covered by PVC sheets or hand gloves, thus minimizing risk of aerosol coming out of these apertures. The presence of PVC sheet fixed at the patient's end restricts the amount of aerosol coming out while the presence of a negative suction port is important in reducing the aerosol content inside the BSB. The BSB is a multi-utility box which can be used by clinicians for endotracheal intubation or bronchoscopy as the need arises. The BSB can be effectively used for the treatment of suspected as well as confirmed COVID-19 patients. Conclusions: We conclude that the BSB is a reusable, multipurpose aerosol safety barrier which can be utilized for multiple procedures of bronchoscopy as well as intubation to help protect HCWs against COVID-19.

Keywords: Bronchoscopy safety box, COVID-19, health-care workers

\section{INTRODUCTION}

COVID-19 is a new pandemic which is spread by SARS-CoV-2 virus and it causes acute respiratory illness leading to death at advanced stage. ${ }^{[1]}$ The disease has spread around the world. At present, the WHO data state that the virus has resulted in $21,938,207$ total confirmed cases and 775,582 deaths (the data will vary with time) ${ }^{[2]}$ As of May 12, 2020, 22,073 cases of COVID-19 among health-care workers (HCWs) from 52 countries had been reported to the WHO.$^{[3]}$ The current evidence shows that COVID-19 virus is primarily transmitted between people through contact routes and respiratory droplets. ${ }^{[4-9]}$ Transmission of the COVID-19 virus can occur by direct contact with infected people and indirect contact with surfaces in the immediate environment or with objects used on the infected person (e.g., stethoscope or thermometer). Bioaerosols are those airborne particles that originate from bacteria, protists, plants, fungi, and animals (living organisms) or viruses that depend on living organisms. In an analysis of 75,465 COVID-19 cases in China, the airborne transmission

\begin{tabular}{|l|l|}
\hline \multicolumn{2}{|c|}{ Access this article online } \\
\hline Quick Response Code: & Website: \\
\hline & www.ijrc.in \\
\hline & \\
\hline
\end{tabular}

was not reported ${ }^{[10]}$ However, in the context of COVID-19, the airborne transmission may be possible in specific circumstances and settings in which procedures or support treatments that generate aerosols are performed; i.e., endotracheal intubation, bronchoscopy, open suctioning, administration of nebulized treatment, manual ventilation before intubation, turning the patient to the prone position, disconnecting the patient from the ventilator, noninvasive positive-pressure ventilation, tracheostomy, and cardiopulmonary resuscitation. The aerosols generated by such patients can infect clinicians, nurses, medical attendants, as well as the near surroundings thus, infecting other patients too. HCWs also may get infected while collecting samples.

Address for correspondence: Dr. Sameer A. Arbat, 275, Central Bazar Road, Ramdaspeth, Nagpur, Maharashtra, India. E-mail: dr.sameerarbat@gmail.com

This is an open access journal, and articles are distributed under the terms of the Creative Commons Attribution-NonCommercial-ShareAlike 4.0 License, which allows others to remix, tweak, and build upon the work non-commercially, as long as appropriate credit is given and the new creations are licensed under the identical terms.

For reprints contact:WKHLRPMedknow_reprints@wolterskluwer.com

How to cite this article: Arbat SA, Chourasia SR. Bronchoscopy safety box and its utility as a barrier in spread of COVID-19 infection. Indian J Respir Care 2021;10:76-81.

Received: $19-09-2020$

Accepted: 08-12-2020

Revised: 06-12-2020 Published: $31-01-2021$ 
Keeping all this in mind, we decided to make a "Bronchoscopy Safety Box" to help combat the aerosol transmission by the infected patients.

\section{Materials and Methods}

The bronchoscopy safety box (BSB) is a see-through box which is conceptualized and designed as there was a need of safety measures to be taken against COVID-19 infection. As all shops were closed due to lockdown imposed by the government to control the spread of COVID-19 virus infection, this box was fabricated in a warehouse. This box can be made with acrylic sheets with cutting equipment. The description of the material used and its design is as follows.

\section{Acrylic and polyvinyl chloride sheets}

This box is made up of acrylic plastic sheets which are transparent, water-resistant, reusable and cleanable material and polyvinyl chloride (PVC) sheets. Acrylic sheets are arranged to make a box [Figure 1] of dimension as follows:

a. Operator side panel: length 22 inches and height of 18 inches (vertically arranged)

b. Left- and right-side panel: length 14 inches and height of 18 inches (vertically arranged)

c. Top panel: length 22 inches and width of 14 inches (horizontally arranged)

d. Front panel for attaching PVC curtains: length 22 inches and height 4 inches.
Design and arrangement of BSB box is shown in Figure 1a and $b$.

\section{Apertures}

This box has two operator apertures, one assistant aperture (at the side of the box) and one bronchoscopy aperture (at the top of the box). Size and position of aperture is shown in Figure 1c and $\mathrm{d}$. The bronchoscopy aperture is closed with a PVC sheet valve. The operator and assistant apertures are covered with a PVC valve which opens and closes with entry and exit of hands in BSB prototype 1. These apertures are covered with hand gloves in BSB prototype 2 [Figure 2].

\section{Dimension of apertures}

a. Two operator apertures: 5 inches each

b. One assistant aperture: 5 inches

c. Suction aperture: $1 \mathrm{~cm}$.

The patient end has PVC sheet curtains to accommodate the shoulders/chest of patients. There is an additional aperture for connecting a negative suction cannula for suctioning the aerosol generated during the procedure.

\section{Instruction for use}

It is recommended to use the BSB in a dedicated bronchoscopy room with a negative pressure arrangement. After wearing personal protective equipment (PPE), the doctor has to take position at the head end of the patient bed. Then, put the

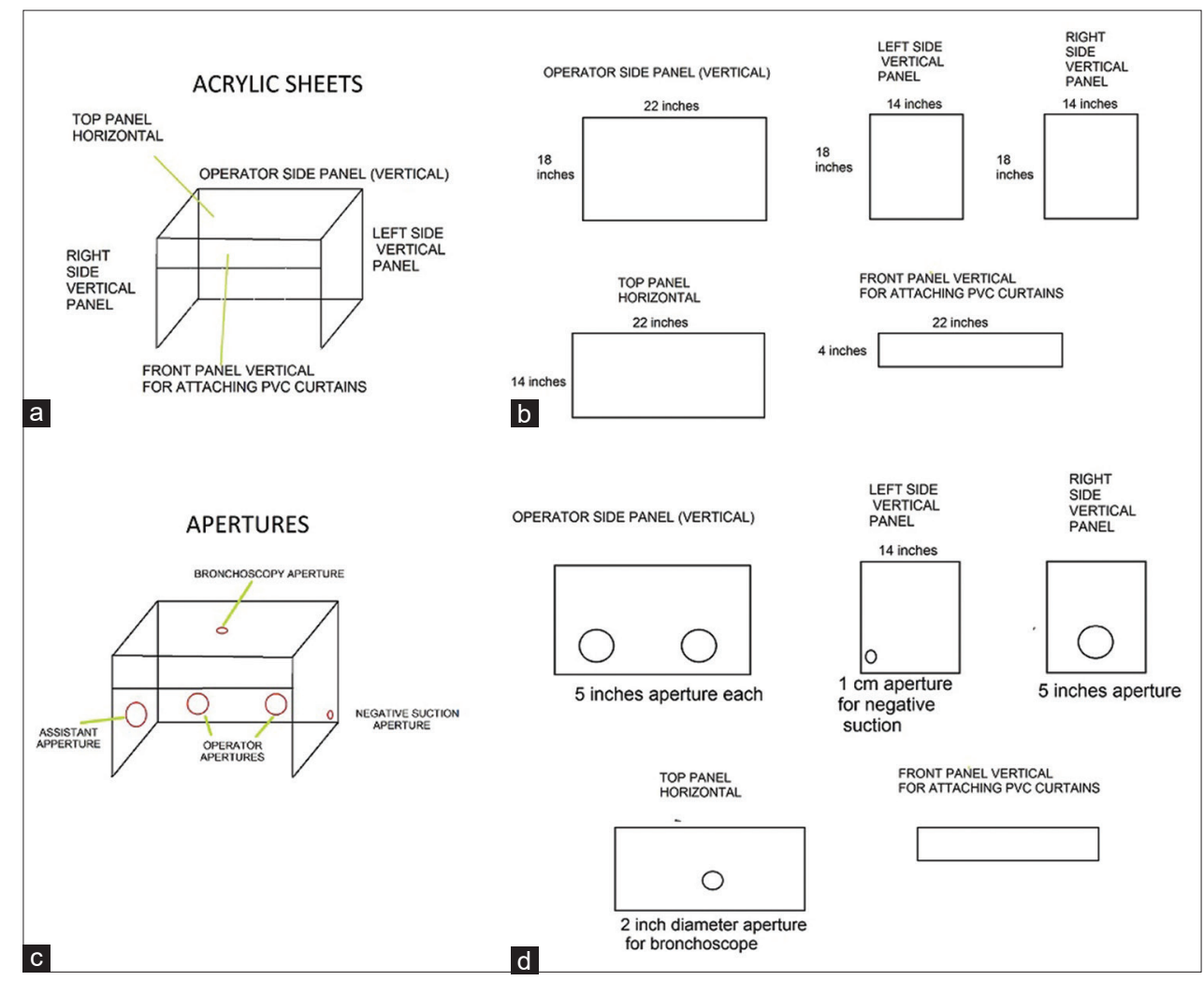

Figure 1: Illustration of bronchoscopy safety box design and material. (a) Structure of bronchoscopy safety box. (b) Dimensions of bronchoscopy safety box. (c) Position of each aperture of the bronchoscopy safety box, namely operator, assistant, bronchoscopy, and negative suction aperture. (d) Size of each aperture 
BSB at the head end of the bed. The patient's head is placed inside the BSB by lifting the PVC curtain. The curtain is then dropped over the patient's neck and chest portion. To perform bronchoscopy, the bronchoscope is inserted inside the box through the bronchoscopy aperture on top. One hand is kept inside the box through the operator aperture to hold the bronchoscope. A doctor performing the bronchoscopy procedure is shown in Figure 3a and 3b.

When endotracheal intubation is to be performed, the intensivist has to put both hands inside the BSB through operator apertures. The procedure of endotracheal intubation is demonstrated in Figure $4 a$ and $b$.

\section{Cleaning of bronchoscopy safety box}

Cleaning of BSB can be done according to local guidelines for disinfection of acrylic and rubbers. BSB can be disinfected with $70 \%$ alcohol or bleach after every use before it is reused for the next patient. Approximately 6 min of negative suction postprocedure is advised to remove all existing aerosols inside the box. Hand gloves can be changed after each procedure and thorough cleaning of BSB.

We used the BSB for 20 procedures, which included 16 bronchoscopy procedures and 4 intubation procedures. By operating the bronchoscope with BSBs, ease of use of BSBs and its limitations were analyzed.

\section{RESULTS}

A forceful cough can generate and spread droplets and aerosols, thus, contaminating the frontline health workers and surrounding. With the use of prototype 1 of BSB, aerosol deposition can be limited to the gloves and sleeves of the PPE of the operator and/or assistant. With the use of prototype 2 of $\mathrm{BSB}$, the contamination can be minimized further as surgical gloves are attached at the apertures. The gloves attached to the operator and assistant aperture are the exclusive barriers that restrict the spread effectively.

We have done twenty procedures using BSB at our center. Out of twenty, sixteen bronchoscopy procedures and four intubation procedures were performed with BSB. All 20 procedures were performed by the specialist and experienced interventional pulmonologist. The patients were COVID-19 negative on reverse transcription-polymerase chain reaction testing. We were able to maneuver the bronchoscope and navigate up to the right lower lobe bronchus and left lower bronchus without any difficulty. The intubation procedure was performed comfortably with the BSB. Ten bronchoscopy procedures were performed with the prototype 1 while six bronchoscopy procedures and four intubations were performed with prototype 2. In all procedures, the assistant aperture proved useful in guiding the operator. The details of the procedures are mentioned in Table 1 . The time taken for foreign body removal took on an average $20 \mathrm{~min}$, whereas, balloon bronchoplasty done for tracheal stenosis took $30 \mathrm{~min}$. Endobronchial ultrasound was

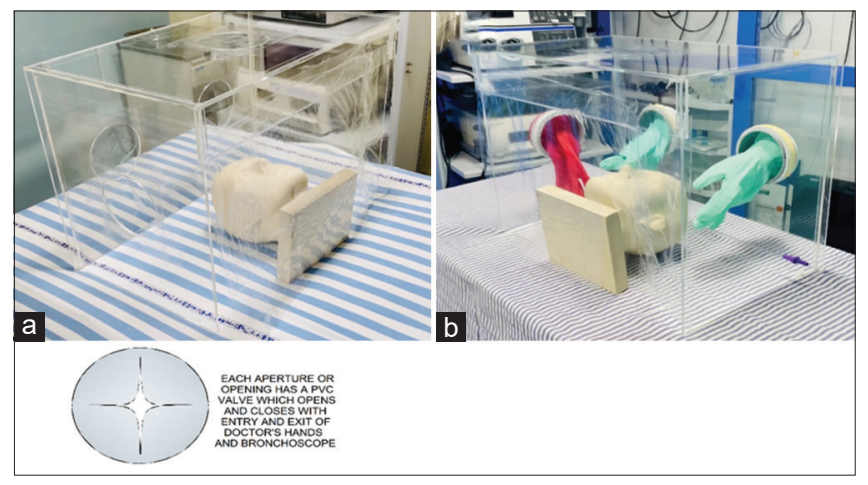

Figure 2: Two prototypes of bronchoscopy safety boxes. (a) Bronchoscopy safety box prototype 1 with polyvinyl chloride sheet valves covering all apertures. Insight picture illustrates the animated design showing the polyvinyl chloride sheet valves. (b) Bronchoscopy safety box Prototype 2 with gloves. Air-tight gloves are attached on all apertures (operator and assistant)
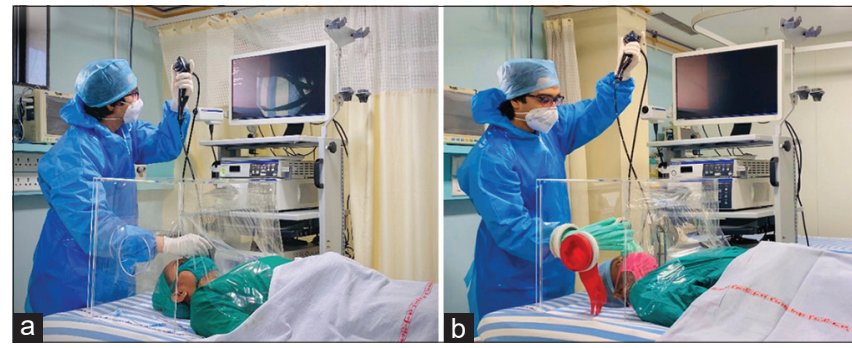

Figure 3: Demonstration of bronchoscopy with bronchoscopy safety box prototype 1 and prototype 2. (a) Demonstration of bronchoscopy with bronchoscopy safety box prototype 1. (b) Demonstration of bronchoscopy with bronchoscopy safety box prototype 2

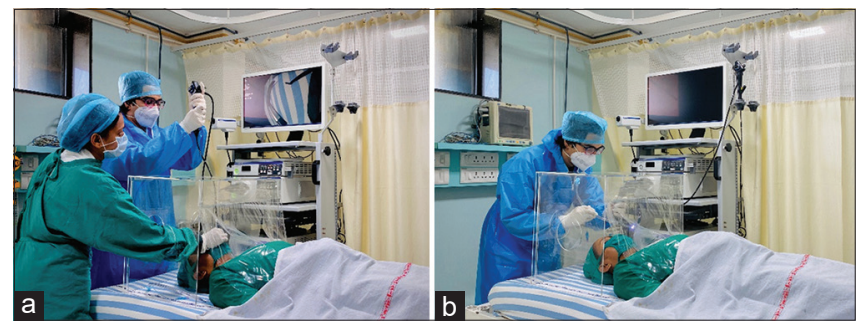

Figure 4: Demonstration of bronchoscopy-guided intubation and endotracheal intubation. (a) Demonstration of bronchoscopy-guided intubation with the use of assistant aperture. (b) Demonstration of endotracheal intubation

performed in $20 \mathrm{~min}$. Bronchial washings and endotracheal intubation were done in 10 and $5 \mathrm{~min}$, respectively. All the procedures were performed with ease using the BSBs with no extra time taken than it usually takes without using BSB. There are several advantages of BSB which are mentioned in Table 2.

There are certain limitations of the BSB which include fixed dimensions of the box that might need some getting used to for operators with different heights and arm lengths. Moreover, while performing difficult intubation, the box might lead to hindrance in using various tools of intubation. 
Arbat and Chourasia: Bronchoscopy safety box: A COVID-19 barrier

\begin{tabular}{|c|c|c|c|c|}
\hline Name of procedure & Number of procedures & RT-PCR for COVID-19 & Indication & Length of procedure (min) \\
\hline Foreign body removal & 3 & Negative & Emergency foreign body removal & Approximate 20 \\
\hline Balloon bronchoplasty & 1 & Negative & Tracheal stenosis & 30 \\
\hline Endobronchial ultrasound & 5 & Negative & Mediastinal lymphadenopathy & 20 \\
\hline Bronchial washings & 7 & Negative & Consolidation & 10 \\
\hline Endotracheal intubation & 4 & Negative & Respiratory failure & 5 \\
\hline
\end{tabular}

RT-PCR - Reverse transcription-polymerase chain reaction

\section{Table 2: Summary of advantages of bronchoscopy safety box}

\begin{tabular}{l}
\hline Advantages of bronchoscopy safety box \\
\hline Bronchoscopy aperture for performing bronchoscopy procedures and \\
EBUS \\
Assistant aperture for guidance \\
All apertures are tightly covered with \\
PVC valves in BSB prototype 1 \\
Gloves in BSB prototype 2 \\
Presence of a negative suction port \\
PVC sheets at the patient end for minimizing aerosol release outside the \\
box \\
Complete box assembly can be disinfected easily with 70\% alcohol or \\
bleach \\
Multipurpose box useful in ICUs for bronchoscopy procedures and \\
intubation \\
Useful in patients of all infective etiologies, e.g., H1N1, COVID-19, \\
MDR-TB, XDR-TB, TB, etc. \\
\hline EBUS - Endobronchial ultrasound, PVC - Polyvinyl chloride, BSB \\
- Bronchoscopy safety box, ICUs - Intensive care units, MDR-TB - \\
Multidrug-resistant tuberculosis, XDR-TB - Extensively drug-resistant \\
tuberculosis, TB - Tuberculosis
\end{tabular}

\section{Discussion}

Physicians are very well aware of the risks associated with respiratory infection that spread while performing aerosol-generating procedures such as bronchoscopy/ intubation of infected patients.

With increasing cases of COVID-19 worldwide, several medical centers are lacking PPE. According to current evidence, COVID-19 virus is primarily transmitted between people through respiratory droplets and contact routes. ${ }^{[4-9]}$ The WHO continues to recommend airborne precautions according to the risk assessment for circumstances and settings in which aerosol-generating procedures and support treatment are performed, for example, endotracheal intubation, bronchoscopy, open suctioning, administration of nebulized treatment, etc. ${ }^{[11]}$ It is advisable for the doctors and staff to wear proper PPE kit for maximum safety. A high mortality has been seen among doctors and health-care providers around the world during the COVID-19 outbreak ${ }^{[12]}$ Hence, clinicians are improvising protective barrier enclosures for use during high-risk treatment procedures like self-sealing openings with viral filter, miniSQUAIR ${ }^{\circledR}$ Aerosol box, etc.

Some groups have come up with innovative solutions that could prove helpful, in case the virus outbreak possesses stiffer challenges. Leading the way in this path, Robert Canelli et al. have come up with an "aerosol box." ${ }^{13]}$ This box acts as a barrier between infected patient and the clinician. They have performed an experiment showing the spread of droplets while performing intubation with and without aerosol box. Without aerosol box, the droplets were scattered in near surroundings, on the mask and PPE of the clinician, hands, bed linen, and as well as on the floor. On the other hand, while performing the same experiment with the aerosol box, the droplets were found only inside the box, on the hand gloves and hands exposed inside the box. Thus, the box helps in restricting the spread of droplets and thus, could help combat the contagion.

There are some limitations to this above-mentioned box such as the aerosol could remain in the air with the COVID-19 virus up to $4 \mathrm{~h}$. Furthermore, these aerosols can come out of the box from the operator's apertures too. We have designed and described the improvised version of such barrier which can overcome the above-mentioned limitation of the aerosol box. Our design can be fabricated as a safety box for performing difficult bronchoscopy procedures in suspected or confirmed COVID-19 patients and may help protect clinicians during this procedure. This box can also be used for endotracheal intubation, which is an important emergency procedure required in patients of COVID-19. ${ }^{[14]}$ The basic prototype of aerosol barrier, described here is "BSB," which consists of a transparent acrylic plastic cube designed to cover a patient's head to the shoulder region. It provides maximum visibility to perform the procedures without any hindrance. It incorporates two circular ports called as operator's aperture through which the clinician's hands are passed to perform the procedure. It has one bronchoscopy aperture at the top of the box with a PVC valve and a lid. For assistance, there is an assistant aperture at the right-side panel. For prevention of droplet and aerosol spread from the infected patient, a PVC curtain is attached at the patient end of the cube. For effective placement, this flap is slit vertically so that it covers the head to shoulder part of the patient like a curtain and there is minimal spread of aerosol from the infected patient. There is negative suction port attached at the left side of the box for suction which is connected directly to the wall-mounted suction port. This added feature further ensures safety against the COVID-19 virus spread among medical staff.

There are two prototypes of BSB developed by us. In Prototype 1, the ports are covered with a PVC sheet valves which open and close with the entry and exit of the clinician's 
hands. The Prototype 2 of BSB has hand gloves attached to it. The operator and assistant aperture are attached with elbow-length hand gloves. When this box is used for the intubation process, then the bronchoscopy aperture can be covered with the acrylic lid.

Any theory or hypothesis needs its authentication and approval in practical terms. Here, we have performed bronchoscopy and intubation with the BSB Prototype 1 and Prototype 2. We were able to maneuver the bronchoscope effectively and navigate up to the left lower bronchus and right lower lobe bronchus without any difficulty. As mentioned in Table 1, we have used the BSB in 20 procedures. 16 bronchoscopy procedures and 4 intubation procedures were performed comfortably with the BSB. The assistant aperture proved useful in guiding the operator during intubation and bronchoscopy (aerosol generating) procedures. The versatility of BSB advances itself to widespread use.

Recently, a clinical demonstration for the BSB was telecast by the Italian Association of Hospital Pulmonologists who have accepted the utility of this box and named it after the inventor. The box has been mentioned in the "Position Paper on Bronchoscopy Role and Modality in COVID-19" published by the Italian Association of Hospital Pulmonologists and Italian Thoracic Society. ${ }^{[15]}$

There are several advantages of using the BSBs. The first advantage is that this box can be utilized for bronchoscopic procedures such as bronchial washing, bronchial alveolar lavage, bronchial brushing, bronchoscopic transbronchial lung biopsy, and endobronchial ultrasound EBUS procedures which can be easily performed with the BSB. The second advantage is that all the apertures are sealed to minimize exit of aerosols out of the box. The ports are covered by the PVC sheets in the BSB prototype 1 and with the hand gloves in BSB prototype 2 .

The third advantage is the utility of the assistant aperture. While performing intubation or bronchoscopy, an assistant can advance his/her hand inside the safety box and help in manipulating the patient's neck or chin for assisting the procedure. The fourth advantage is the presence of PVC sheet fixed at the patient's end which restricts the amount of aerosol coming out of the box from the patient end. The fifth advantage is the presence of a negative suction port which is important in reducing the aerosol content inside the BSB. The sixth advantage is that BSB is a multi-utility box. Clinicians in the intensive critical care units can use it for endotracheal intubation or bronchoscopy as per requirement. This box can be effectively used for the treatment of suspected as well as confirmed COVID-19 patients, cases of any bacterial or viral infectious diseases such as H1N1, tuberculosis (TB), multidrug-resistant TB, extensively drug-resistant TB. The box is easy to disinfect with $70 \%$ alcohol or bleach and can be used for more than 100 patients. The box has a simple design which is easy to fabricate and should cost around INR 5000 or 60 American Dollars approximately.
We would like to advise the clinicians to follow the specific guidelines on the use of bronchoscopy in patients with suspected or confirmed COVID-19 infection. ${ }^{[16]}$ The main purpose of this innovative box is to ensure the safety of patients and health-care team.

There are certain limitations to this study. In our study, the number of patients is less as we are following the guidelines issued by AABIP which states that bronchoscopy should be performed only in emergency cases. With a greater number of patients, this study will further enlighten its utility and limitations in practical terms. Another limitation of the BSB includes fixed dimensions of the box which might need some getting used to for operators with different heights and arm lengths. The third limitation states that while performing difficult intubation, the box might lead to hindrance in using various tools of intubation. Other than this, both the BSBs are useful and we believe that this system can help further by decreasing exposure to health-care professionals and save their lives. We recommend medical professionals should perform procedures with BSBs under specific guidelines to ensure minimal COVID-19 infection.

\section{Conclusions}

We conclude that the BSB is a reusable, multipurpose aerosol safety barrier which can be utilized for multiple procedures of bronchoscopy as well as intubation to help protect HCWs against COVID-19.

\section{Acknowledgment}

We thank Dr. Stefano Gasparini - Director, Specialty School in Respiratory Medicine Pulmonary Diseases Unit, Azienda Ospedaliero-Universitaria "Ospedali Riuniti" Via Conca, 71-60020 Ancona-Italy, for his technical advice, thoughtful input, and for critically reviewing this study proposal.

\section{Financial support and sponsorship}

Nil.

\section{Conflicts of interest}

There are no conflicts of interest.

\section{References}

1. Lu R, Zhao X, Li J, Niu P, Yang B, Wu H, et al. Genomic characterisation and epidemiology of 2019 novel coronavirus: Implications for virus origins and receptor binding. Lancet 2020;395:565-74.

2. WHO Coronavirus Disease (COVID-19) Dashboard. Available from: https://covid19.who.int. [Last accessed 2021 Jan 09].

3. Coronavirus Disease 2019 (COVID-19) Situation Report-82. Available from:https://www.who.int/docs/default-source/coronaviruse/ situation-reports/20200411-sitrep-82-covid-19.pdf? sfvrsn=74a5d15_2. [Last accessed on 2020 Apr 11].

4. Liu J, Liao X, Qian S, Yuan J, Wang F, Liu Y, et al. Community transmission of severe acute respiratory syndrome coronavirus 2, Shenzhen, China, 2020. Emerg Infect Dis 2020;26:1320-3.

5. Chan JF, Yuan S, Kok KH, To KK, Chu H, Yang J, et al. A familial cluster of pneumonia associated with the 2019 novel coronavirus indicating person-to-person transmission: A study of a family cluster. Lancet 2020;395:514-23.

6. Li Q, Guan X, Wu P, Wang X, Zhou L, Tong Y, et al. Early transmission 
dynamics in Wuhan, China, of novel coronavirus-infected pneumonia. N Engl J Med 2020;382:1199-207.

7. Huang C, Wang Y, Li X, Ren L, Zhao J, Hu Y, et al. Clinical features of patients infected with 2019 novel coronavirus in Wuhan, China. Lancet 2020;395:497-506.

8. Burke RM, Midgley CM, Dratch A, Fenstersheib M, Haupt T, Holshue $\mathrm{M}$, et al. Active monitoring of persons exposed to patients with confirmed COVID-19 - United States, January-February 2020. MMWR Morb Mortal Wkly Rep 2020;69:245-6.

9. World Health Organization. Report of the WHO-China Joint Mission on Corona virus Disease 2019 (COVID-19) 16-24 February 2020. Geneva: World Health Organization; 2020. Available from: https://www.who. int/docs/default-source/coronaviruse/ who-china-joint-mission-on-covid-19-final-report.pdf. [Last accessed on 2020 Feb 28].

10. Ong SWX, Tan YK, Chia PY, Lee TH, Ng OT, Wong MSY, et al. Air, surface environmental, and personal protective equipment contamination by severe acute respiratory syndrome coronavirus 2 (SARS-coV-2) from a symptomatic patient. JAMA 2020;323:1610-2.

11. WHO Infection Prevention and Control Guidance for COVID-19. Available from: https://www.who.int/emergencies/ diseases/novel-coronavirus-2019/technical-guidance/ infection-prevention-and-control. [Last accessed 2020 Sep 11].

12. Italian Doctors' Fatalities Reach Tragic Levels as they Fight COVID-19 in Overburdened Hospitals. Available from: https://www. theglobeandmail.com/world/article-italian-doctors-fatalities-reach-tragi c-levels-as-they-fight-covid-1/. [Last accessed on 2020 Apr 03].

13. Canelli R, Connor CW, Gonzalez M, Nozari A, Ortega R. Barrier enclosure during endotracheal intubation. N Engl J Med 2020;382:1957-8.

14. Luo M, Cao S, Wei L, Tang R, Hong S, Liu R, et al. Precautions for intubating patients with COVID-19. Anesthesiology 2020;132:1616-8.

15. Role and Methods of Performing Bronchoscopy in the COVID-19 Pandemic-AIPO Position Paper. Available from: http://www.aiponet.it/ component/attachments/download/2637.html. [Last accessed on 2020 Apr 17].

16. Wahidi M, Lamb C, Murgu S, et al. American Association for Bronchology and Interventional Pulmonology (AABIP) statement on the use of bronchoscopy and respiratory specimen collection in patients with suspected or confirmed COVID-19 infection. AABIP website. https://aabronchology.org/wp-content/uploads/2020/03/2020-AABIPStatement-on-Bronchoscopy-COVID.GAE-updated-Version.pdf. Published March 2020. [Last accessed on 2020 Apr 24]. 